\title{
Big Bang theory
}

\author{
Half of the 2019 Nobel Prize in Physics was awarded to James Peebles "for theoretical discoveries in physical \\ cosmology" and the other half is shared by Michel Mayor and Didier Queloz "for the discovery of an exoplanet \\ orbiting a solar-type star".
}

W

hen we look at the night sky, we see not only the Moon but hundreds or thousands of stars - maybe even one of the brighter planets in our Solar System. It's hard to grasp that this visible matter makes up a mere five per cent of our Universe. With the remainder thought to be dark matter and dark energy, we seem to know little about the Universe. However, to honour the knowledge that we have, this year's Nobel Prize in Physics was awarded to James Peebles, Michel Mayor and Didier Queloz "for [their] contributions to our understanding of the evolution of the Universe and Earth's place in the cosmos".

An early indication for the origin of our Universe was the suggestion by Robert Dicke, James Peebles, Peter Roll and David Wilkinson that the cosmic microwave background could originate from a hot Big Bang ${ }^{1}$. But this would not remain Peebles's only important contribution to modern cosmology. Throughout his career, many more publications ranging from the cosmic microwave background to galaxy formation followed.
One of his most notable works, carried out jointly with Jeremiah Ostriker, was the discovery that large amounts of dark matter must be present in the halo of spiral galaxies such as the Milky Way as otherwise the flat galactic disk would be unstable ${ }^{2}$. In 1982, Peebles's studies of non-relativistic or cold dark matter (CDM; ref. ${ }^{3}$ ) laid the foundations for the standard model of cosmology - the $\Lambda \mathrm{CDM}$ model. Apart from standard baryonic matter, this model incorporates CDM and dark energy associated with the cosmological constant $\Lambda$, which Peebles put back on the map after its famous dismissal by Albert Einstein.

Whereas Peebles shaped our current understanding of how galaxies and galaxy clusters form, Mayor's and Queloz's discovery influenced our knowledge of planet formation. When the two started their monitoring campaign, planets outside our Solar System (exoplanets) orbiting a pulsar had been discovered, but not in orbit around a solar-type star. Periodic variations in the radial velocity of the star 51 Pegasi revealed such a planetary companion, 51 Pegasi b (ref. ${ }^{4}$ ). The exoplanet's mass was estimated to be at least half of Jupiter's - a puzzling observation in light of the short orbital period of around four days.

The discovery of 51 Pegasi b posed a riddle to planet formation as its separation from 51 Pegasi was too small for the planet to be Jupiter-like. The authors speculated that the exoplanet might have been formed from a stripped brown dwarf. Since Mayor's and Queloz's observations, thousands of exoplanets have been discovered and continue to inspire advances in planetary formation models.

Despite this huge leap in understanding of our Universe, plenty of discoveries are still waiting to be made - from the exact process of planet formation to figuring out what dark matter is made of. Whatever we'll find along the way, we know that it all started with the Big Bang.

Published online: 4 November 2019 https://doi.org/10.1038/s41567-019-0720-4

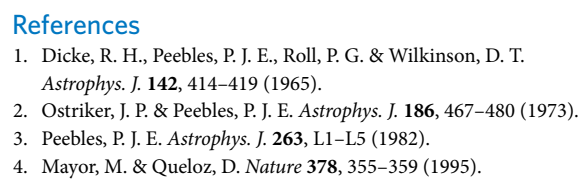

\section{A supreme achievement}

\section{The demonstration of a quantum computational advantage is a milestone worth celebrating.}

\section{L}

ast month, Nature published one of the worst kept secrets in physics: the experimental demonstration of 'quantum supremacy' reported by a collaboration led by the Google AI Quantum team.

The feat was achieved by Sycamore, a quantum processor made up of 53 superconducting circuits operating below $20 \mathrm{mK}$, which took 3 minutes to carry out a calculation that is estimated to take 10 millennia on our best classical supercomputers. It consists of sampling from the output distribution of a random quantum circuit, a task with essentially no other purpose than demonstrating a quantum computational advantage.

Google AI Quantum's team had been pursuing this goal for some time. For many specialists in the field, it was a matter of when it would happen, rather than if. Nevertheless, the wider reaction to the report, first broken by the Financial Times in September after a preprint of the paper was accidentally posted on a public NASA server, was one of huge excitement. Pronouncements that we are at the dawn of a new technological era abounded, and for a short while many scientists felt uncomfortable, as often happens when a story gets too hot to handle.

More critical voices therefore quickly appeared to manage expectations, pointing out the relatively arbitrary nature of the threshold that constitutes quantum supremacy, and cautioning against the potential corporate interests behind a loud announcement with little practical substance. If quantum and classical machines are pitted against each other on a task that has been engineered to favour the former, the argument goes, should we really take this to be such a meaningful display of superiority? Outperforming classical machines on an actually meaningful task surely ought to be the real goal, and that's likely still a long way off.

But there is little doubt that Google's experiment represents an extraordinary feat of physics, computer science and engineering. If only as a good excuse to pause and celebrate the journey that took us from the dawn of quantum information science in the 1980s all the way to performing computations by navigating a Hilbert space of $2^{53}$ dimensions, the achievement is well worth the title of a scientific milestone.

And as with all good milestones, quantum supremacy is really just the beginning. A few decades from now we may well acknowledge that this was, after all, a fairly arbitrary threshold. But it is one that seems destined to inspire a future generation of scientists.

Published online: 4 November 2019 https://doi.org/10.1038/s41567-019-0721-3 